\title{
Barriers to Surgical Care Among Children in Somaliland: An Application of the Three Delays Framework
}

\author{
Tessa L. Concepcion ${ }^{1} \cdot$ Shukri Dahir $^{2} \cdot$ Mubarak Mohamed $^{2} \cdot$ Kyle Hiltbrunn $^{3}$ • \\ Edna Adan Ismail ${ }^{2} \cdot$ Dan Poenaru $^{4} \cdot$ Henry E. Rice ${ }^{1} \cdot$ Emily R. Smith $^{1,3}$ on behalf of the \\ Global Initiative for Children's Surgery
}

Published online: 6 February 2020

(C) Société Internationale de Chirurgie 2020

\begin{abstract}
Background There are complex barriers that increase delays to surgical care in low- and middle-income countries, particularly among the vulnerable population of children. Understanding these barriers to surgical care can result in targeted and strategic intervention efforts to improve care for children. The three-delay model is a widely used framework in global health for evaluating barriers associated with seeking (D1), reaching (D2), and receiving health care (D3). The goal of our study is to evaluate reasons for delays in the surgical care for children in Somaliland using the three-delay framework.

Methods Data were collected in a cross-sectional study in Somaliland from 1503 children through a household survey. Among children with a surgical need, we quantified the number of children seeking, reaching, and receiving care along the surgical care continuum, according to the three-delay framework. We evaluated predictors of the three delays through a multivariate logistic regression model, including the child's age, gender, village type, household income level, region, and household size.

Results Of the 196 children identified with a surgical condition, $50(27.3 \%)$ children had a delay in seeking care (D1), $28(20.6 \%)$ children had a delay in reaching care (D2), and $84(71.2 \%)$ children had a delay in receiving care (D3), including 10 children who also experienced D1 and D2. The main reasons cited for D1 included seeking a traditional healthcare provider, while lack of money and availability of care were main reasons cited for D2. Significant predictors for delays included household size for D1 and D3 and condition type and region for D2. Conclusion Children in Somaliland experience several barriers to surgical care along the entire continuum of care, allowing for policy guidance tailored to specific local challenges and resources. Since delays in surgical care for children can substantially impact the effectiveness of surgical interventions, viewing delays in surgical care under the lens of the three-delay framework can inform strategic interventions along the pediatric surgical care continuum, thereby reducing delays and improving the quality of surgical care for children.
\end{abstract}

Emily R. Smith

Emily_R_Smith@baylor.edu

1 Duke Global Health Institute, Duke University, Durham, NC, USA

2 Edna Adan University Hospital, Hargeisa, Somaliland

3 Department of Public Health, Robbins College of Health and Human Sciences, Baylor University, 1621 S. 5th Street, Waco, TX 76706, USA

4 McGill University, Montreal, QC, Canada

\section{Introduction}

Surgical delivery in low- and middle-income countries (LMICs) is increasingly recognized as essential to health system strengthening and universal health coverage (UHC) [1-3]. However, there are complex barriers that increase delays to surgical care in LMICs, namely limited accessibility and availability, particularly among the vulnerable population of children who are unable to advocate on their 
own behalf. A child who lives hours away from a healthcare facility capable of performing surgery does not have access to safe and timely surgical care. On the other hand, simply living in close proximity to a healthcare facility does not ensure adequate surgical care for that child if there is a shortage of surgeons and anesthetists or lack of essential supplies. Thus, accessibility and availability are critical, distinct concepts in improving surgical care for children in LMICs across the care continuum. Understanding these barriers to surgical care can result in targeted and strategic intervention efforts to improve care for children.

The three-delay model is a widely used framework in many areas of global health, including surgery, to evaluate barriers associated with seeking, reaching, and receiving health care [4, 5]. Although the framework can help delineate barriers to care and guide targeted interventions, the framework has not been applied specifically to children's surgical care for an entire low-income country. Each delay outlined in the three-delay framework plays a vital role in the timely and proper treatment of surgical conditions in children, particularly when the conditions are associated with high mortality or lifelong morbidity if not treated early. In addition, the consequences of delaying surgical care for children include presentation with advanced stages of disease, resulting in higher rates of untreatable morbidities. Use of the three-delay framework for evaluation of surgical systems for children may aid policy makers in prioritizing specific interventions to improve access to high-quality care.

Our previous work has identified a high burden of surgical conditions in children across Somaliland, substantial impact on poverty dynamics, as well as lack of surgical infrastructure and workforce, particularly in rural areas [6-8]. Building on this work, the goal of our current study is to identify reasons for delays in seeking or receiving surgical care for children in Somaliland according to the three-delay framework.

\section{Methods}

\section{Setting}

Our study took place in Somaliland, a country in the Horn of Africa, which, although not recognized as an independent state, has achieved relative stability since separation from Somalia and has had an autonomous government since 1991. The country is classified as a low-income country by the World Bank income groups, with a gross domestic product (GDP) per capita of $\$ 348$, ranking the country as the fourth poorest in the world [9]. Infant and under-5 mortality rates are over twice as high as most areas in sub-Saharan Africa, and approximately $50 \%$ of the total population of 4 million people are under the age of 16 years [10-12]. Somaliland includes six regions: Awdal, Maroodi Jeex, Sahil, Sanaag, Sool, and Togdheer. Among those living in rural areas, 38\% are living in poverty, compared with $29 \%$ in urban areas [13]. In extreme poverty, the disparity is even more pronounced, with $24 \%$ of residents in rural areas living in extreme poverty, compared to $8 \%$ in urban areas [13].

The public health system in Somaliland is comprised of public facilities (primary health units, health centers, regional hospitals, and one national referral hospital) and private facilities, including specialty hospitals. Surgical care for children is concentrated within urban areas, with a limited number of procedures at mainly private hospitals [8].

\section{Survey sampling and data collection}

Our current study is an extension of previous work examining the burden of pediatric surgical conditions in children, economics, and hospital infrastructure and workforce in Somaliland [6]. In 2017, we surveyed 1503 children ages $0-15$ years from all six regions of Somaliland to measure the prevalence of surgical conditions using the Surgeons OverSeas Assessment of Surgical Need (SOSAS) tool, and the methodology has been previously described [6]. Briefly, survey clusters throughout Somaliland were randomly selected in a two-stage process with a probability adjustment for population size. We collected data on household demographic characteristics and surgical condition history on all children in the household using a cross-sectional survey by a team of Somali data collectors who visited the houses. Demographic characteristics included household location (region), village type, household size, and number of children within the household. We also collected information regarding the family's assets and impact on poverty trends as described elsewhere [7]. For this purpose, we queried each household for the assets they owned using the Social Capital Assessment Tool (SCAT) [14]. Assets included chickens, farm animals, TV/ radio, refrigerator, washing machines, and cars and were totaled for a final asset score.

We defined surgical conditions using the Lancet Commission on Global Surgery (LCoGS) as "any disease, illness, or injury in which surgical care can potentially improve the outcome" [15]. Surgical need was self-reported by the parents or guardians of the children as a condition that required surgical consultation. Care provided by a traditional healer, defined as care provided outside of a healthcare facility, was not considered as healthcare seeking. If health care was sought at a hospital, respondents also reported the type of health care received for the 
condition as: (1) not receiving health care nor surgery, (2) receiving health care but not surgery, (3) receiving a minor procedure, or (4) receiving a major procedure.

\section{Barriers to surgical care and the three-delay framework}

Barriers to seeking and/or receiving surgical care for the children with surgical conditions were provided through self-report by the survey respondent and included financial restrictions, lack of transportation, fear/lack of trust, care not available, or a perceived lack of need by the respondent. Respondents could cite more than one barrier to seeking or receiving care.

We quantified the number of children seeking, reaching, and receiving surgical care along the surgical care continuum using the three-delay framework [4]. The first delay (D1) — the delay in seeking care-was defined as seeking care from a traditional healer prior to seeking formal care at a healthcare facility, or not seeking any care due to a lack of perceived need, fear, or no time to seek care. The second delay (D2) - the delay in reaching care-was defined as not seeking care due to financial constraints or unavailable healthcare infrastructure. The third delay (D3) - the delay in receiving care-was defined as reaching formal health care at a health facility, but not receiving surgical care.

\section{Data analysis}

Data were analyzed using SAS 9.4 (SAS, Cary, NC) and Microsoft Excel 2010 (Microsoft, Redmond, WA). Demographic characteristics, including child's age and sex, were compared across regions using the Wald Chi-squared statistic for categorical variables and $t$ test statistics for continuous variables. Healthcare seeking behavior, the receipt of surgical intervention, and barriers to each of these constructs were compared across regions using proportions rather than the Wald Chi-squared statistic, due to small sample sizes.

We evaluated significant predictors of each stage of the three-delay framework through a multivariate logistic regression model, including the child's age, gender, village type, household income level, region, and household size. To improve model robustness, variables were collapsed into several categories. Condition type was categorized into wounds, congenital deformities, and other conditions (including burns, acquired deformities, masses, and gastrointestinal problems); asset count was categorized as 0 assets, 1-2 assets, and 3 or more assets; region was categorized as Maroodi Jeex/Sahil, indicating urban regions, Awdal/Sool/ Sanaag, and Togdheer; household size was categorized as 3-5 family members, 6-10 members, and 11 or more members; and number of children within the household was dichotomized to $1-3$ children and 4 or more children. Statistical significance for all results was evaluated at $P<0.05$.

\section{Ethics statement}

Institutional review board (IRB) approval was granted from Duke University. Since Somaliland does not have a national IRB, a letter of approval was granted from the Somaliland Ministry of Health. Participants in the community survey offered verbal consent for study participation. A parent or guardian provided consent for all children younger than 15 years old, and children between the ages of 12 and 15 provided additional assent.

\section{Results}

We found substantial delays in seeking, reaching, and receiving surgical care among children in Somaliland. The predictors of delays in care were found in all stages of the surgical care continuum using the three-delay framework.

Of the 196 children with a surgical condition, 50 children (27.3\%) had a delay in seeking care (D1), 28 children $(20.6 \%)$ had a delay in reaching care (D2), including 3 children who also had a delay in seeking care, and a total of 84 children $(71.2 \%)$ had a delay in receiving care (D3), including 10 children who also experienced D1 and D2 (Fig. 1). The main reasons cited for the D1 were seeking a traditional healthcare provider $(n=47)$ and lack of perceived need $(n=16)$ (Table 1$)$. Of the 28 children with D2, the main reasons cited included lack of money for health care and transportation $(n=22)$. Of the 84 children with D3, the main reasons for delaying receiving care included no perceived need $(n=33)$, lack of money for transportation $(n=23)$, and lack of available healthcare facility, personnel, and/or equipment $(n=11)$.

Significant predictors of not seeking surgical care (D1) included the number of children within the household (Fig. 2). Households with more than 3 children were 4.8 times more likely to not seek care $(95 \%$ CI $1.4,16.6)$ than household with 3 or fewer children. Significant predictors of not reaching care (D2) included condition type and region. Children suffering from burns, acquired deformities, masses, and gastrointestinal problems were 9.9 times more likely to delay reaching care $(95 \%$ CI $1.8,53.8)$ than children with surgical conditions of wounds. Children with surgical conditions living in the rural regions of Awdal, Sool, or Sanaag were 4.4 times more likely to delay reaching care $(95 \%$ CI $1.1,18.0)$ than children within surgical conditions living in the urban regions of Maroodi Jeex or Sahil. Significant predictors of reaching care but 


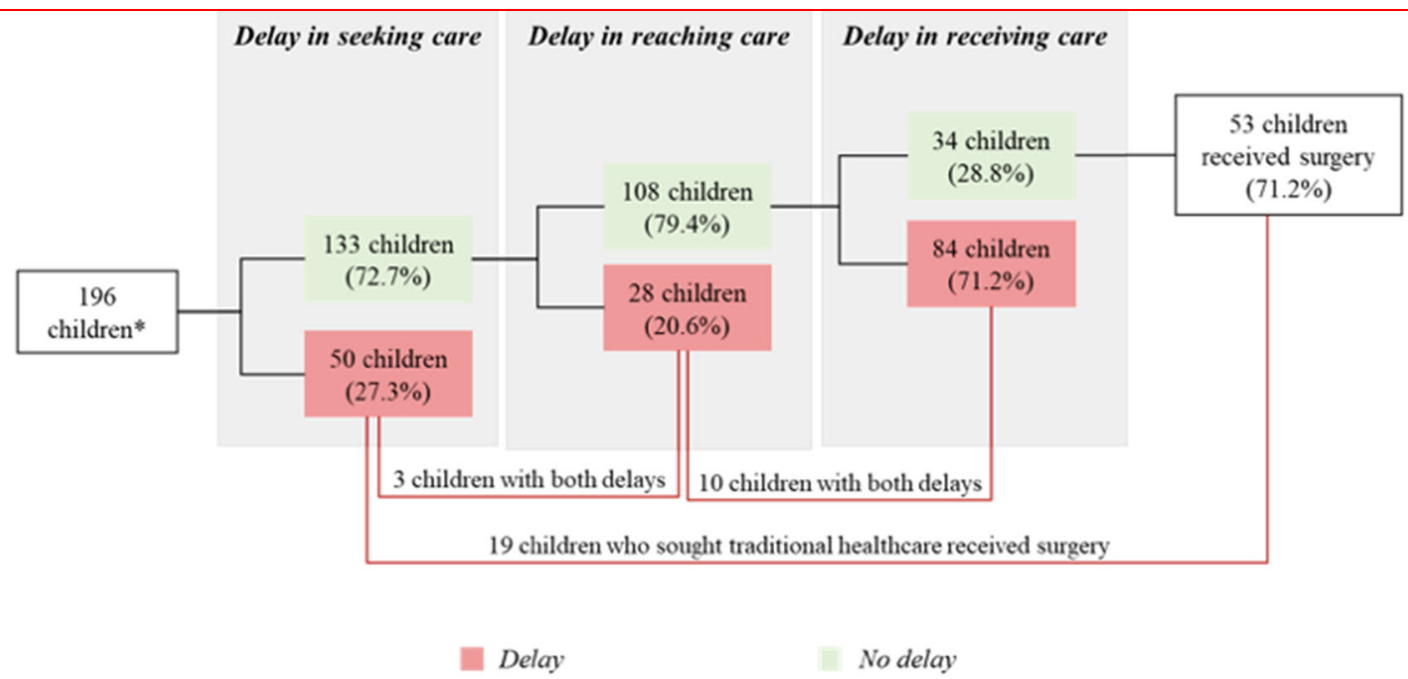

* 9 children had conditions with unknown healthcare/surgical status; 5 children had conditions with an unknown delay. 23 children had multiple conditions. Of these children, 12 had the same delay for both conditions and 11 had different delays for both conditions. When omitting duplicates randomly, we lose 6 "delay in seeking", 2 "delay in reaching", 1 "delay in receiving" and 4 conditions with no delays.

Fig. 1 Reasons for not receiving surgery $(n=196)$. Note: D1, delay in seeking care; D2, delay in reaching care; D3, delay in receiving care

Table 1 Reasons for delaying seeking care, reaching care, and receiving care

\begin{tabular}{lccc}
\hline & $\begin{array}{l}\text { Delay in seeking } \\
\text { care (D1, } n=50)\end{array}$ & $\begin{array}{l}\text { Delay in reaching } \\
\text { care (D2, } n=28)\end{array}$ & $\begin{array}{l}\text { Delay in receiving } \\
\text { care (D3, } n=84)\end{array}$ \\
\hline No time & 2 & & 33 \\
Fear/lack of trust & 6 & & 10 \\
No perceived need & 16 & 3 & 23 \\
Sought traditional health care & 47 & 20 & 11 \\
No money for health care & & 2 & 17 \\
No money for transportation & & 6 & \\
Health care unavailable & & & \\
No reason cited & & & \\
\hline
\end{tabular}

${ }^{a}$ Not available includes facility, personnel, and/or equipment

not receiving surgical care (D3) included asset count and household size. Children living in homes with lower assets were 2.3 times more likely to delay receiving care $(95 \% \mathrm{CI}$ $0.7,7.8)$ than those with higher asset counts. Children living in homes with large families were also more likely to delay receiving care from a healthcare facility, even when care was sought.

\section{Discussion}

Children in Somaliland have barriers to surgery along the entire continuum of care. Viewing these delays through the three-delay framework allows for identification of interventions to reduce delays in seeking antenatal care and reaching or receiving needed obstetric care [4]. This framework has been applied to define barriers to maternal health as well as surgical care for general populations in LMICs [5], but has not specifically been used to measure delays in surgical care for children, who have unique preoperative, perioperative, and postoperative needs. Since delays in surgical care for children can substantially impact the effectiveness of surgical interventions [16-19], viewing delays in surgical care under the lens of the three-delay framework can inform strategic interventions along the pediatric surgical care continuum, thereby reducing delays at all levels.

Preventing the first two delays in seeking and reaching care involves a functional and coordinated health system comprised of an interdependent network of individuals, 
Fig. 2 Odds of delays for seeking, reaching, and receiving surgical care for children in Somaliland

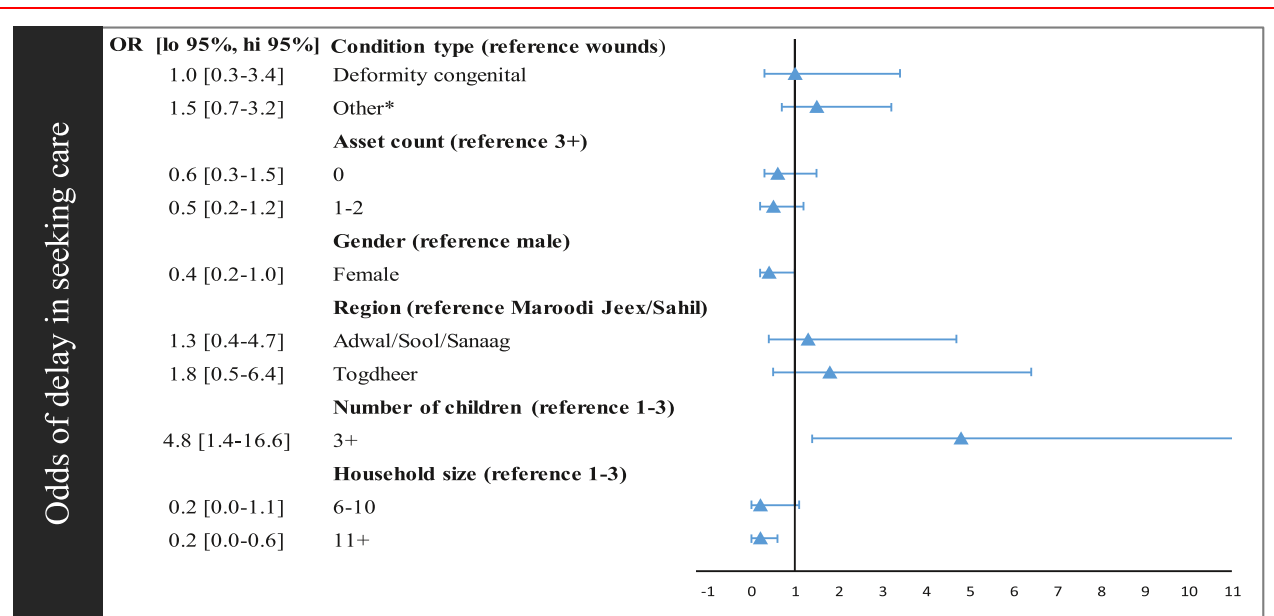

OR [lo 95\%. hi 95\%] Condition type (reference wounds)

$1.8[0.1-41.7] \quad$ Deformity congenital

$9.9[1.8-53.8] \quad$ Other*

Asset count (reference 3+)

$1.4[0.3-6.3] \quad 0$

$1.9[0.3-11.3] \quad 1-2$

Gender (reference male)

$1.3[0.7-2.4] \quad$ Female

Region (reference Maroodi Jeex/Sahil)

$4.4[1.1-18.8] \quad$ Adwal/Sool/Sanaag

$1.1[0.2-8.1] \quad$ Togdheer

Number of children (reference 1-3)

$1.2[0.1-9.4] \quad 3+$

Household size (reference 1-3)

$0.8[0.2-3.6] \quad 6-10$

$0.2[0.0-4.1] \quad 11+$

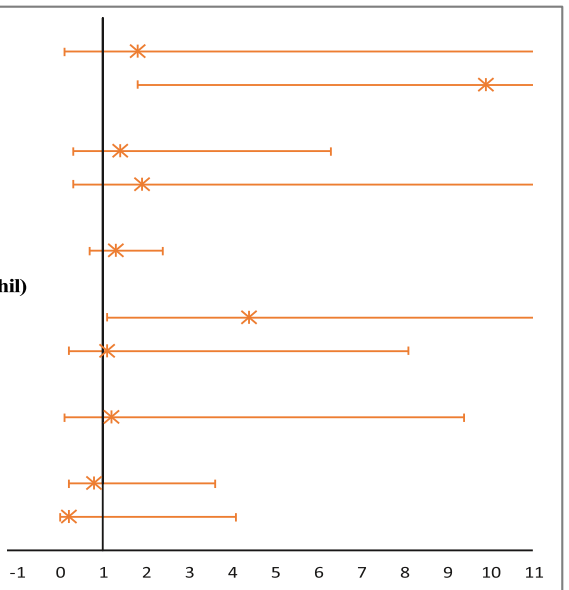

OR [lo 95\%. hi 95\%] Condition type (reference wounds)

$0.9[0.2-3.9]$ Deformity congenital

$0.8[0.3-1.9] \quad$ Other*

$2.3[0.7-7.8] \quad 0$

$1.8[0.8-4.0] \quad 1-2$

Gender (reference male)

$1.4[0.6-3.0] \quad$ Female

Region (reference Maroodi Jeex/Sahil)

$0.7[0.2-1.8] \quad$ Adwal/Sool/Sanaag7

$1.2[0.3-4.2] \quad$ Togdheer

Number of children (reference 1-3)

$0.6[0.3-1.3] \quad 3+$

Household size (reference 1-3)

$2.5[1.1-5.8] \quad 6-10$

$3.7[1.3-10.6] \quad 11+$

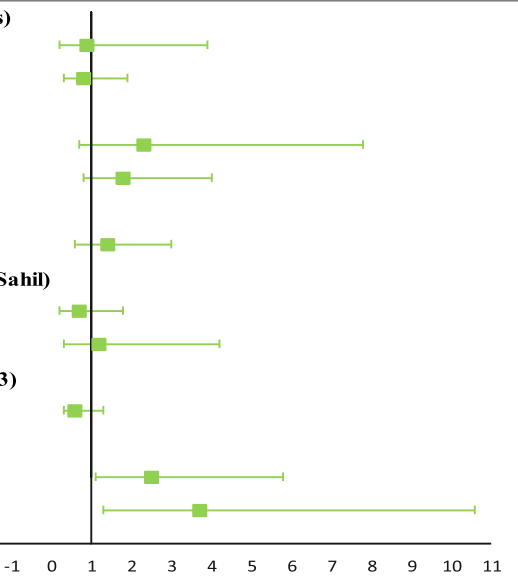

*Other $=$ burns, acquired deformities, masses, and GI problems

facilities, and delivery infrastructures. At the community level, health workers can connect patients to first-level hospitals, who can provide most essential and emergent procedures. In turn, first-level hospitals should refer complex cases to higher levels of care at secondary or tertiary centers for specialized care. This shared surgical ecosystem supports the timely, affordable, and safe receipt of surgical care at all levels of care. From a system level, a strong surgical ecosystem includes engaging community health workers and informal providers, especially in rural areas. We confirmed that significant barriers to seeking care in Somaliland include cultural beliefs, fear, and lack of time. On an individual level, monetary constraints played a strong role among families experiencing a delay in reaching care such as transportation to facilities that can be hours or days away [20-29]. These data suggest that an enhanced 
system of community-based surgical referrals may improve access to high-quality surgical care. Similar systems have been tested for adults in Bangladesh with the Bangladesh Rural Advancement Committee, who has systematically reduced the first and second delays through equipping community health workers to identify complicated cases and coordinate timely and reliable transportation to a higher-level facility [30].

From a healthcare infrastructure perspective, Somaliland has limited surgical capacity for children in the country, particularly in rural areas, driving delays along the firstand second-delay framework. More than half of the hospitals are located in the capital city of Hargeisa, while the more rural areas have one hospital for the entire region [8]. We found that even among families who were able to reach a hospital, care was not available. Even if the facilities had a fully functional surgical system, families who cannot afford paying for portions or all of the procedures may experience a delay in receiving care, as nearly $50 \%$ of families in our study cited financial constraints as the most significant barrier to receiving care [7]. These findings suggest that specific interventions to improve local surgical infrastructure and manpower, as well as programs to reduce user fees and other expenses, may improve access to care.

Although interventions to reduce the first and second delays can improve many areas of surgical care, reducing the third delay from a system standpoint is arguably the most complex for the surgical care of children. Many complicated surgical conditions for children require care which can only be provided at tertiary-level or national hospitals. Many of these conditions have extremely high mortality rates if not operated on promptly. These children require specific pediatric resources, such as specialized anesthesia, supplies, and equipment, suggesting that reducing the third delay requires bolstering up infrastructure and manpower at each level of the health system as outlined by the Global Initiative for Children's Surgery [31, 32].

Our study has several limitations. Our data were collected in a cross-sectional manner through self-report from family caregivers of children with surgical conditions. Due to the retrospective nature of data collection, we were unable to assess the severity of the surgical condition upon presentation, the quality of operative and postoperative care provided, and long-term follow-up of the children. These factors could have impacted our results if children with more severe conditions died prior to the time of the survey, biasing our findings toward the null. All self-reported data have a risk of recall bias and could have underestimated the proportions of families reporting barriers to care along the surgical care continuum if they underreported reasons for not seeking, reaching, or receiving care. We attempted to minimize the risk of recall bias in our study by hiring and training local, in-country data collectors to conduct all surveys in the household with no study team members from high-income countries present. Our assumption in this approach was to create a level of trust between study participants and enumerators.

\section{Conclusion}

Investing in the surgical care of children in LMICs is costeffective and critical [33, 34], and one of the first steps to scale-up access is understanding reasons for delays in care. Delays in seeking, reaching, and receiving surgical care for children in Somaliland are common, complex, and distinct along the surgical continuum of care. By applying the three-delay framework, we identified reasons for delays at each timepoint, which in turn could guide implementation strategies.

Acknowledgements We want to thank the Global Initiative for Children's Surgery (GICS) for its support of this work. GICS (www. globalchildrenssurgery.org) is a network of children's surgical and anesthesia providers from low-, middle-, and high-income countries collaborating for the purpose of improving the quality of surgical care for children globally.

Funding Funding was provided by Duke Global Health Institute, Duke University and Baylor University.

\section{References}

1. Canfin P et al (2013) Our common vision for the positioning and role of health to advance the UN development agenda beyond 2015. Lancet (London England) 381:1885-1886

2. Kim JY, Farmer P, Porter ME (2013) Redefining global healthcare delivery. Lancet (London England) 382:1060-1069

3. Makasa EM (2014) Letter to global health agency leaders on the importance of surgical indicators. Lancet (London England) 384:1748

4. Thaddeus S, Maine D (1994) Too far to walk: maternal mortality in context. Soc Sci Med (1982) 38(8):1091-1110

5. Kakembo N, Godier-Furnemont A, Nabirye A et al (2019) Barriers to pediatric surgical care in low-income countries: the Three Delays' impact in Uganda. J Surg Res 242:193-199

6. Concepcion $\mathrm{T}$ et al (2019) Prevalence of pediatric surgical conditions across Somaliland. JAMA Netw Open 2:e186857. https:// doi.org/10.1001/jamanetworkopen.2018.6857

7. Smith ER et al (2019) The contribution of pediatric surgery to poverty trajectories in Somaliland. PLoS ONE 14:e0219974. https://doi.org/10.1371/journal.pone.0219974

8. Concepcion TL et al (2019) Provision of surgical care for children across Somaliland: challenges and policy guidance. World J Surg 43:2934-2944. https://doi.org/10.1007/s00268-019-05079-8

9. New World Bank GDP and Poverty Estimates for Somaliland. World Bank. http://www.worldbank.org/en/news/press-release/ 2014/01/29/new-world-bank-gdp-and-poverty-estimates-forsomaliland. Accessed 16th Nov 2016

10. World Bank (2013) Africa development indicators 2012/2013. World Bank, Washington, DC 
11. You D et al (2015) Global, regional, and national levels and trends in under-5 mortality between 1990 and 2015, with scenario-based projections to 2030: a systematic analysis by the UN Inter-agency Group for Child Mortality Estimation. Lancet (London) 386:2275-2286

12. WHO I Infant mortality. WHO. http://www.who.int/gho/child health/mortality/neonatal_infant_text/en/. Accessed 26th Feb 2017

13. The World Bank (2015) SOMALILAND: poverty profile and overview of living conditions (poverty global practice: Africa region), vol 55. The World Bank, Washington, DC

14. Krishna A (2017) The broken ladder: the paradox and potential of India's one-billion. Cambridge University Press, Cambridge. https://doi.org/10.1017/9781108235457

15. Meara JG et al (2015) Global surgery 2030: evidence and solutions for achieving health, welfare, and economic development. Lancet (London, England) 386:569-624. https://doi.org/10.1016/ s0140-6736(15)60160-x

16. Smith ER, Concepcion T, Lim S, Sadler S, Poenaru D, Saxton AT, Shrime M, Ameh E, Rice HE (2018) Disability weights for pediatric surgical procedures: a systematic review and analysis. World J Surg 42(9):3021-3034. https://doi.org/10.1007/s00268018-4537-6

17. Magee WP, Vander Burg R, Hatcher KW (2010) Cleft lip and palate as a cost-effective health care treatment in the developing world. World J Surg 34(3):420-427. https://doi.org/10.1007/ s00268-009-0333-7

18. Wang H, Bhutta ZA, Coates MM, Coggeshall M, Dandona L, Diallo K, Franca EB, Fraser M, Fullman N, Gething PW, Hay SI (2016) Global, regional, national, and selected subnational levels of stillbirths, neonatal, infant, and under-5 mortality, 1980-2015: a systematic analysis for the Global Burden of Disease Study 2015. Lancet (London, England) 388(10053):1725-1774

19. Smith ER, Concepcion TL, Shrime M, Niemeier K, Mohamed M, Dahir S, Ismail EA, Poenaru D, Rice HE (2019) Waiting too long: the contribution of delayed surgical access to pediatric disease burden in Somaliland. World J Surg. https://doi.org/10. 1007/s00268-019-05239-w

20. Abdelgadir J, Punchak M, Smith ER et al (2018) Pediatric traumatic brain injury at Mbarara Regional Referral Hospital, Uganda. J Clin Neurosci Off J Neurosurg Soc Australas 47:79-83

21. Abdelgadir J, Smith ER, Punchak M et al (2017) Epidemiology and characteristics of neurosurgical conditions at Mbarara regional referral hospital. World Neurosurg 102:526-532

22. Appenteng R, Nelp T, Abdelgadir J et al (2018) A systematic review and quality analysis of pediatric traumatic brain injury clinical practice guidelines. PLoS ONE 13(8):e0201550
23. Bearden A, Fuller AT, Butler EK et al (2018) Rural and urban differences in treatment status among children with surgical conditions in Uganda. PLoS ONE 13(11):e0205132

24. Butler EK, Tran TM, Nagarajan N et al (2017) Epidemiology of pediatric surgical needs in low-income countries. PLoS ONE 12(3): 0170968

25. Fuller AT, Haglund MM, Lim S et al (2016) Pediatric neurosurgical outcomes following a neurosurgery health system intervention at Mulago Hospital in Uganda. World Neurosurg 95:309-314

26. Smith ER, van de Water BJ, Martin A et al (2018) Availability of post-hospital services supporting community reintegration for children with identified surgical need in Uganda. BMC Health Serv Res 18(1):727

27. Smith ER, Vissoci JRN, Rocha TAH et al (2017) Geospatial analysis of unmet pediatric surgical need in Uganda. J Pediatr Surg 52(10):1691-1698

28. Butler EK, Tran TM, Fuller AT et al (2016) Quantifying the pediatric surgical need in Uganda: results of a nationwide crosssectional, household survey. Pediatr Surg Int 32(11):1075-1085

29. Davé DR, Nagarjan N, Canner JK, Kushner AL, Stewart BT, SOSAS4 Research Group (2018) Rethinking burns for low \& middle-income countries: differing patterns of burn epidemiology, care seeking behavior, and outcomes across four countries. Burns 44(5):1228-1234

30. Jolly SP, Rahman M, Afsana K, Yunus FM, Chowdhury AM (2016) Evaluation of maternal health service indicators in urban slum of Bangladesh. PLoS ONE 11(10):e0162825

31. Global Initiative for Children's Surgery (2019) Optimal resources for children's surgical care: executive summary. World J Surg 43(4):978-980. https://doi.org/10.1007/s00268-018-04888-7

32. Global Initiative for Children's Surgery (2019) A model of global collaboration to advance the surgical care of children. World J Surg 43(6):1416-1425. https://doi.org/10.1007/s00268-01804887-8

33. Smith ER, Concepcion TL, Niemeier KJ, Ademuyiwa AO (2019) Is global pediatric surgery a good investment? World J Surg 43:1450-1455. https://doi.org/10.1007/s00268-018-4867-4

34. Saxton AT et al (2016) Economic analysis of children's surgical care in low- and middle-income countries: a systematic review and analysis. PLoS ONE 11:e0165480. https://doi.org/10.1371/ journal.pone.0165480

Publisher's Note Springer Nature remains neutral with regard to jurisdictional claims in published maps and institutional affiliations. 